\title{
Design and development of Binary Diffractive Germanium Lens by thin film deposition
}

\author{
M. Alshami \\ manal.alshami@hiast.edu.sy
}

\section{A. Wabby}

\section{F. Mousselly}

Higher Institute for Applied Sciences and Technology Damascus, Syria

Higher Institute for Applied Sciences and Technology Damascus, Syria

Higher Institute for Applied Sciences and Technology Damascus, Syria

The design and development of infrared $(\lambda:[8]-[12] \mu \mathrm{m})$ binary diffractive germanium lens (BDGL) by two - steps thin film deposition (Physical vapor deposition (PVD) technique) is presented. The optical design of the required elements using the optical design code Zemax, the design of the 4 steps binary surface and its required metallic masks using the programming language Delphi, the procedures of fabrication, and the measurement of the resulting profile, were presented. The comparison between the refractive/diffractive lenses by measuring the minimum resolvable temperature difference (MRTD) shows the advantages of binary diffractive surface.

[DOI: http://dx.doi.org/10.2971/jeos.2015.15055]

Keywords: Diffractive, binary surface, thin film

\section{INTRODUCTION}

The use of diffractive optical elements can be traced back to Lord Rayleigh, who, in 1871, was apparently the first to make use of the device known today as the Fresnel zone plate (FZP) [1]. The action of a diffractive lens is based on near field diffraction at a FZP [2].

A high diffraction efficiency can be achieved by implementing the FZP pattern as a blazed phase structure, as it was proposed by Dammann in 1970 [3], and recent developments in the fabrication of high efficiency diffractive optical elements, known as kinoforms [4], binary optics [5], or phase Fresnel lenses [6], have generated a renewed interest in diffractive optics. The fabrication techniques, e.g., precision diamond machining [7], photolithography [8], and laser writer systems [9], provide the designer with the ability to choose a desired diffractive phase function, however, the fabrication of phase profile, with a continuous depth, is a cost technology [10]. The theoretical ideal profile of the diffractive surface can be approximated in a discrete fashion, similar to the digital representation of an analog function [10]. This discrete representation is called a multi-level or binary. The primary method of fabricating such diffractive elements has been reactive ion etching of a multi-level surface relief grating on one side of a lens [11], where a diffractive element imparts a phase delay to an incident wavefront in a very thin layer close to the surface of the element [10]. The thickness of this layer is on the order of the incident wavelength. The phase delay is imparted to the incident wavefront by selectively removing material from the surface of the substrate [11], or adding material to the surface of the substrate using Lithography [12] or thin film deposition (coating) [2], of the same substrate's material, where the fabrication of diffractive lenses using the deposition of thin dielectric films was first demonstrated by L. D'Auria [13]. The fabrication of binary diffractive lenses by thin film deposition needs special masks, which can be produced by coating the substrate with a film of a photoresist using standard optical lithography, then transfer the mask pattern into the resist, but the disadvantage of this method is the need for high cleanness, where the presence of some particles of photoresist in the undesired places causes instability of the thin film. In this paper instead of using photoresist masks, we used metallic masks to avoid this problem, which have the same diameter as the substrate. The use of metallic masks is suitable to the infrared spectrum, where the longer the wavelength the wider the diffractive zones.

Hopefully, this paper will provide the reader with some insights into the potential usefulness of using metallic masks and thin film coating to fabricate binary (multi-level) diffractive phase profiles in the infrared spectrum, which can be easily designed and evaluated along with the procedures detailed in this paper. The fabrication tools and equipment necessary to produce these elements are not expensive, and they are standard equipments and are used in the fabrication of thin film coating.

The first part of the paper presents the optical design using the optical design program Zemax of the two (Plano - convex) refractive and diffractive germanium lenses. The second part describes the technological processes to fabricate the BDGL by two steps thin film deposition (PVD), the measurement of the resulting profile, and the comparison, where the criterion to compare the two refractive/diffractive germanium lenses 
was the measurement of the MRTD of the two lenses under the same conditions.

\section{OPTICAL DESIGN IN ZEMAX}

Diffractive lenses are essentially gratings with variable groove spacing across the optical surfaces, which impart a change in phase of the wavefront passing through the surface. The optical design program ZEMAX [14], does not model the wavelength - scale grooves directly. Instead, ZEMAX uses the phase advance or delay represented by the surface locally to change the direction of propagation of the ray.

Diffractive surface are defined in ZEMAX by the Binary Optic 2 surface, where The Binary Optic 2 surface adds a symmetrical phase to the ray according to the following polynomial expansion [14]:

$$
\varnothing=\mathrm{M} \sum_{\mathrm{i}=1}^{\mathrm{N}} \mathrm{A}_{\mathrm{i}} \rho^{2 \mathrm{i}}
$$

Where $\mathrm{N}$ is the number of polynomial coefficients in the series, $\mathrm{A}_{i}$ is the coefficient on the $2 \mathrm{i}^{\text {th }}$ power of $\rho$ which is the normalized radial apertures coordinate, and $\mathrm{M}$ is the diffraction order. The more terms are used, the more complicated the structure of the binary surface is. Here we only used the first two terms. The primary procedure is as follows:

\subsection{Optical design of a refractive germanium (Plano - convex) lens}

Optical design of a refractive germanium (Plano - convex) lens using Zemax, for the wavelength band [8]-[12] $\mu \mathrm{m}$ is presented in Figure 1, Table 1. It has an effective focal length of $75 \mathrm{~mm}$ with a $9.09^{\circ}$ field of view according to the detector's dimensions $(384 \times 288$ pixels with pixel's size $25 \mu \mathrm{m})$, which will be used in the measurement of the MRTD, the diameter of the lens is $33 \mathrm{~mm}$

\subsection{Optical design of the diffractive germanium lens}

Optical design of the diffractive germanium lens using Zemax with the same conditions as refractive one, and choose the plane surface as the binary 2 surface, as mentioned above we only used the first two terms of the phase with the first diffraction order in Eq. (1), and define them as variables in the extra data editor, keeping the effective focal length unchanged, as shown in Figure 2, Table 2.

$$
\begin{aligned}
\varnothing & =\mathrm{A}_{1} \rho^{2}+\mathrm{A}_{2} \rho^{4} \\
A 1 & =-0.65554, \\
A 2 & =8.97588
\end{aligned}
$$

Figure 3, shows the MTF (up to 10 cycles/mm, Nyquist frequency) [15] curves of the refractive/diffractive lenses in the same field of view. The RMS radius values of the spot of the refractive/diffractive lenses in the same field of view are in Table 3. Comparing the RMS radius values in Table 3 and the MTFs curves of the two lenses, we can see that the optical performance of the diffractive lens is clearly better than the refractive one especially for the axial field of view.

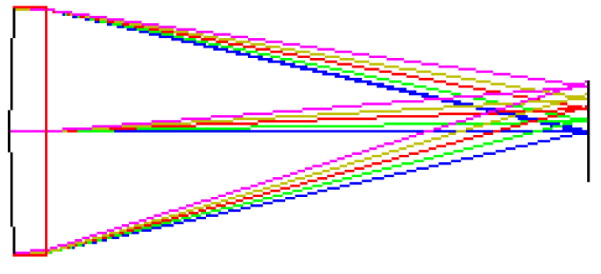

FIG. 1 Layout of the refractive lens.

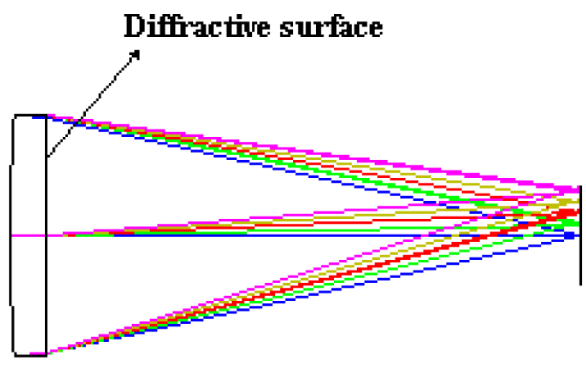

FIG. 2 Layout of the diffractive lens.

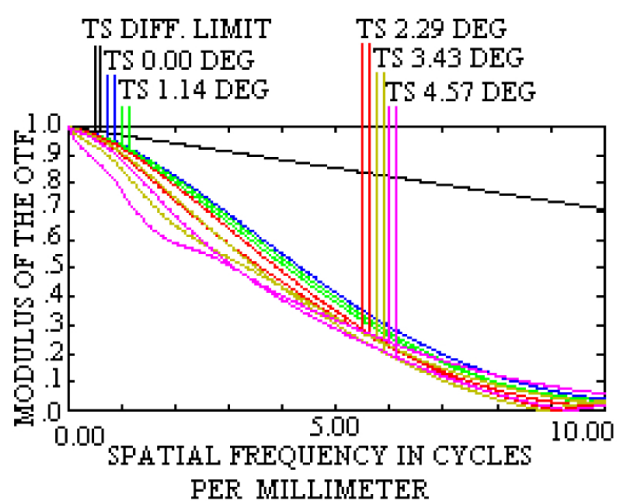

(a)

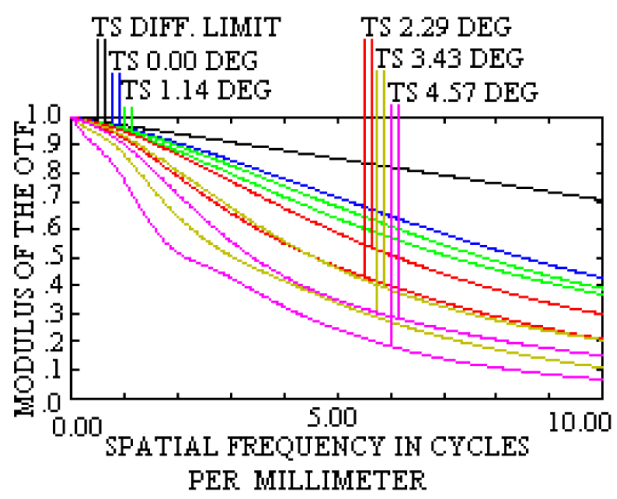

(b)

FIG. 3 The MTF curves of the refractive lens (a) and the diffractive lens (b).

\section{FABRICATION OF THE BINARY OPTICAL LENS}

The first step involved in fabricating a multi-level element is to mathematically describe the ideal diffractive phase profile that is to be approximated in a multi-level fashion and to generate from it a set of masks that contains the phase profile information. In our case, the mathematical phase is expressed 


\begin{tabular}{|c|c|c|c|c|c|}
\hline Surface & Type & Radius & Thickness & Glass & Diameter \\
\hline OBJ & Standard & Infinity & Infinity & & 0.000 \\
\hline STO & Standard & 225.371 & 5.000 & Germanium & 33.049 \\
\hline 2 & Standard & Infinity & 72.849 & & 32.652 \\
\hline IMA & Standard & Infinity & & & 6.846 \\
\hline
\end{tabular}

TABLE 1 Specifications of the refractive lens $(\mathrm{mm})$.

\begin{tabular}{|c|c|c|c|c|c|}
\hline Surface & Type & Radius & Thickness & Glass & Diameter \\
\hline OBJ & Standard & Infinity & Infinity & & 0.000 \\
\hline STO & Standard & 225.371 & 5.000 & Germanium & 33.050 \\
\hline 2 & Binary2 & Infinity & 73.339 & & 32.657 \\
\hline IMA & Standard & Infinity & & & 6.901 \\
\hline
\end{tabular}

TABLE 2 Specifications of the diffractive lens $(\mathrm{mm})$.

\begin{tabular}{|c|c|c|c|c|c|}
\hline Field Of View (degree) & 0.00 & 1.14 & 2.29 & 3.43 & 4.57 \\
\hline RMS radius of refractive lens(micron) & 84.88 & 100.99 & 140.88 & 194.02 & 257.81 \\
\hline RMS radius of diffractive lens(micron) & 41.16 & 66.03 & 114.03 & 170.69 & 235.78 \\
\hline
\end{tabular}

TABLE 3 RMS radius values of the refractive/diffractive germanium lenses.

using Eq. (2):

$$
\varnothing=-0.656 \rho^{2}+8.976 \rho^{4}
$$

The maximum number of periods of binary surface $\mathrm{n}_{\max }$ is given by:

$$
\mathrm{n}_{\max }=\operatorname{Int}\left|\left(-0.656 \rho^{2}+8.976 \rho^{4}\right) / 2 \pi\right|
$$

Where Int denotes the integration operation. In our design, there is one period on the binary surface. Apparently by controlling $A_{1}$ and $A_{2}$ in the optimization, there might be fewer periods on the binary surface, which means a bigger line width beneficial to manufacturing. Figure 4 shows the phase vs. aperture of the diffractive surface.

The next step in the fabrication process, once the phase function is mathematically determined, is to create a set of metallic masks which are produced by laser $\mathrm{CO} 2$ machine (with a fabrication accuracy $0.1 \mathrm{~mm}$ ), to create these masks some calculations must be done, which Zemax isn't sufficient for doing, for that calculations, a Delphi's code was written to calculate the diameter of each discrete phase level or binary zone, Table 4 , then the design of the required masks according to the number of that levels. In the practical work the number of those levels was 4, as Figure 5 shows.

The last step in the fabrication process is thin film deposition. The relation between the maximum depth of every diffractive zone, the design wavelength, and the index of refraction of the lens material is given by Eq. (5) [11]:

$$
\mathrm{d}_{\mathrm{opt}}=\lambda /(\mathrm{n}-1)
$$

Where $\lambda$ is the central wavelength of the spectral band of interest ( $\lambda=10$ microns), $\mathrm{n}$ is index of refraction (For the material germanium $\mathrm{n}=4.00312$ for $\lambda=10$ microns), then: $\mathrm{d}_{\text {opt }}=3.33$ microns, so the thickness of the first layer to be deposited with the first mask by thin film deposition is $1.667 \mathrm{mi}-$ cron (equivalent to phase $\pi$ ), and the thickness of the second layer with the second mask is 0.833 micron (equivalent to phase $\pi / 2$ ).

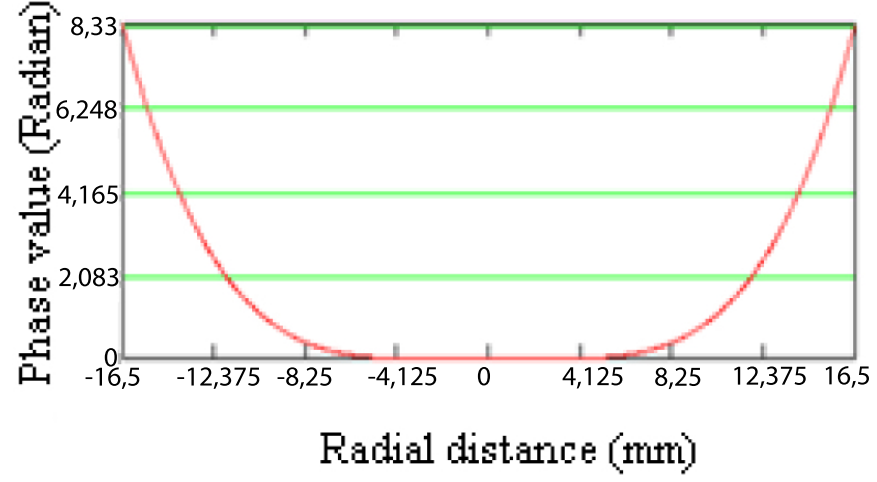

FIG. 4 Phase curve vs. aperture of the diffractive surface.

In order to deposit the first germanium layer, Figure 6, the first metallic mask is placed in intimate contact with the substrate (germanium lens) and the both are placed in a suitable metallic holder (with fabrication accuracy $0.01 \mathrm{~mm}$ ) in the PVD apparatus (which is electron-beam gun) and the temperature of the evaporation chamber set to $\left(140^{\circ} \mathrm{C}\right)$. The metallic holder will control the alignment's accuracy of the substrate and the first mask.

After the deposition of the first germanium layer (Thickness 1.667 micron) by the PVD apparatus, Figure 7, the germanium lens is removed from the PVD apparatus, and then the second metallic mask is placed in intimate contact with the substrate and the both are placed in the same metallic holder in the PVD apparatus and the temperature of the evaporation chamber set to $\left(140^{\circ} \mathrm{C}\right)$, then the deposition of the second germanium layer (Thickness 0.833 micron) is done.

The metallic holder also controlled the alignment of the second mask with the substrate. When the thin film were deposited by two-step with the two metallic masks which have a fabrication accuracy $0.1 \mathrm{~mm}$, an expected error in the width of each binary zone will result, as shown in Figure 7. 


\begin{tabular}{|c|c|c|c|}
\hline $\begin{array}{c}\text { The binary zone's } \\
\text { number }\end{array}$ & $\begin{array}{c}\text { The first diameter of } \\
\text { the binary zone }(\mathrm{mm})\end{array}$ & $\begin{array}{c}\text { The second diameter } \\
\text { of the binary zone }(\mathrm{mm})\end{array}$ & $\begin{array}{c}\text { The width of the } \\
\text { binary zone }(\mathrm{mm})\end{array}$ \\
\hline 1 & 22.295 & 26.177 & 1.941 \\
\hline 2 & 26.177 & 30.851 & 2.337 \\
\hline 3 & 28.807 & 30.851 & 1.022 \\
\hline
\end{tabular}

TABLE 4 Diameters and width of each binary zone.

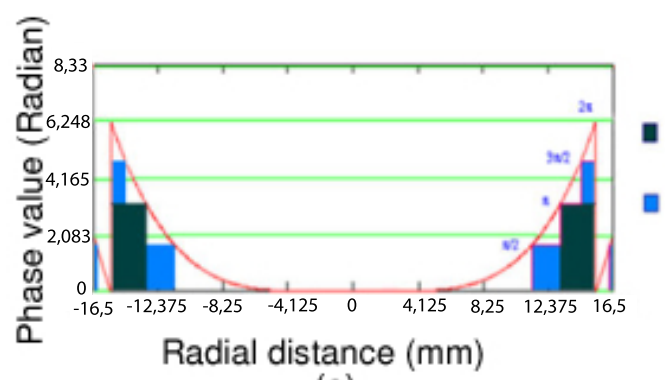

(a)
Thin film deposition with mask 1

\section{Thin film deposition with mask 2}

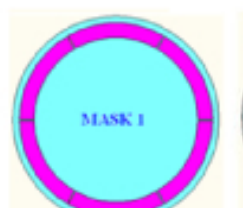

(b)

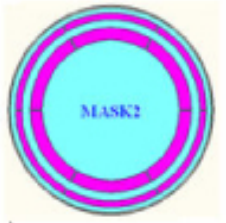

FIG. 5 Phase curve vs. aperture of the binary surface slicing into $2 \pi$ layers and the discrete phase levels (a) and the required metallic masks (b).

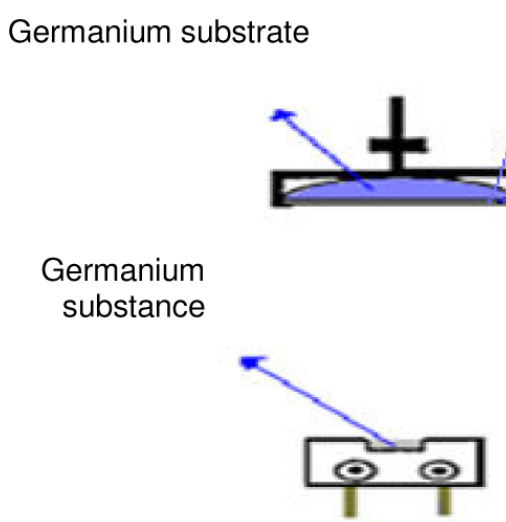

FIG. 6 Schematic drawing of the thin film deposition process.

Figure 8 shows a part of the resulting sag profile of the BDGL, which has been measured by the Surface roughnessmeasuring instrument (SE-3400) in a room with temperature of $20^{\circ} \mathrm{C}$. As shown in Figure 8, The resulting profile didn't keep rectangular shoulders and changed roundish shape due to the thickness of the two metallic masks ( $1 \mathrm{~mm}$ per mask), which causes a small error $\Delta \mathrm{d}$ from the continuous sag profile ( $\Delta \mathrm{d} \approx 0.067$ microns), Figure 8 . This small error has no effect on the diffraction efficiency, where the diffraction efficiency of discrete phase levels binary structure related just to the number $\mathrm{N}$ of the discrete levels at the design wavelength [10], Eq. (6):

$$
\eta^{\mathrm{N}}=[\sin (\pi / \mathrm{N}) /(\pi / \mathrm{N})]^{2}
$$

From Eq. (6), the diffraction efficiency of 4 phase levels binary structure at the design wavelength is 0.81 . From other side the maximum depth $\mathrm{d}_{\text {opt }}=3.33$ microns, so an error of $0.067 \mathrm{mi}$ crons expressed as a fraction of the total depth is e $=0.02$ relates to a reduction of the diffraction efficiency (for the continuous profile) of less than 1, which can be found from Eq. (7) [11]:

$$
\eta 1=[\sin (\pi \mathrm{e}) /(\pi \mathrm{e})]^{2}
$$

The theoretical germanium The theoretical germanium

layer obtained with the

layer obtained with the

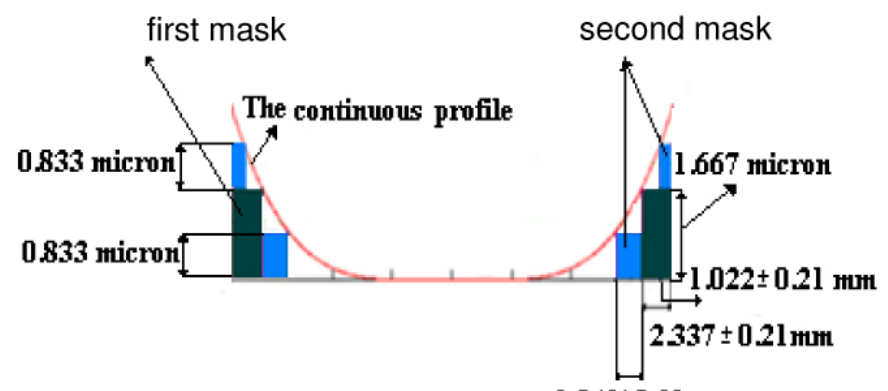

$1941 \pm 0.21 \mathrm{muth}$

FIG. 7 Germanium layers (binary zones) and the expected error in its width.

\section{COMPARISON BETWEEN THE BINARY DIFFRACTIVE GERMANIUM LENS AND THE REFRACTIVE ONE}

The MRTD is a subjective parameter that describes ability of the imager-human system for detection of low contrast details of the tested object [16].

Generally, MRTD is measured by determining the minimum temperature difference between the bars of the standard 4-bar target and the background required to resolve the thermal image of the bars by an observer for 4-bar targets of different dimensions (spatial frequency).

MRTD is a measure of ability to detect and recognize targets on non-uniform background.

The MRTD values are calculated for each observer from recorded positive temperature difference $\left(\Delta \mathrm{T}_{+}\right)$and negative temperature difference $\left(\Delta \mathrm{T}_{-}\right)$using the formula [16]:

$$
\operatorname{MRTD}=\left[\left(\Delta \mathrm{T}_{+}-\Delta \mathrm{T}_{-}\right) / 2\right]
$$




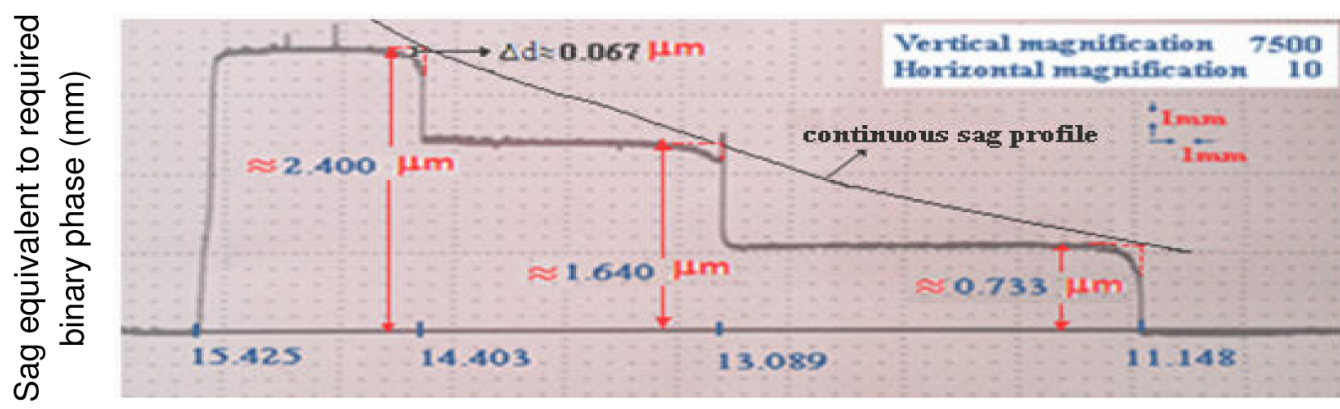

Radial distance $(\mathrm{mm})$

FIG. 8 The continuous sag profile and a part of the resulting profile of the BDGL.

\begin{tabular}{|c|c|c|c|}
\hline $\begin{array}{c}\text { Frequency } \\
\text { (cycles/mmradian) }\end{array}$ & $\begin{array}{c}\text { Positive temperature } \\
\text { difference }\left(\Delta \mathrm{T}_{+}\right)\end{array}$ & $\begin{array}{c}\text { Negative temperature } \\
\text { difference }\left(\Delta \mathrm{T}_{-}\right)\end{array}$ & MRTD $\left({ }^{\circ} \mathrm{C}\right)$ \\
\hline 0.000 & 1.5 & -2.5 & 2.00 \\
\hline 0.500 & 3.3 & -3.5 & 3.40 \\
\hline 1.000 & 7.0 & -8.0 & 7.50 \\
\hline 1.695 & 30.0 & -35.0 & 32.50 \\
\hline
\end{tabular}

TABLE 5 MRTD values of the refractive germanium lens.

\begin{tabular}{|c|c|c|c|}
\hline $\begin{array}{c}\text { Frequency } \\
\text { (cycles/mmradian) }\end{array}$ & $\begin{array}{c}\text { Positive temperature } \\
\text { difference }\left(\Delta \mathrm{T}_{+}\right)\end{array}$ & $\begin{array}{c}\text { Negative temperature } \\
\text { difference }\left(\Delta \mathrm{T}_{-}\right)\end{array}$ & MRTD $\left({ }^{\circ} \mathrm{C}\right)$ \\
\hline 0.000 & 1.4 & -2.0 & 1.70 \\
\hline 0.500 & 3.0 & -2.8 & 2.90 \\
\hline 1.000 & 6.0 & -6.5 & 6.25 \\
\hline 1.695 & 30.0 & -26.0 & 28.00 \\
\hline
\end{tabular}

TABLE 6 MRTD values of the binary diffractive germanium lens.

The measured MRTD's of the both refractive/diffractive lenses are listed in Table 5, Table 6, respectively. Their variations with spatial frequencies are plotted in Figure 9. We can see that the BDGL is better in detection (Low frequencies) and recognition (Middle frequencies) than the refractive lens.

\section{CONCLUSIONS}

We designed and fabricated a 4 steps binary diffractive germanium lens with $9.09^{\circ}$ field of view. The MTF performance is better than that of the refractive one, especially in axial field of view. An attempt has been made to show that the process of thin film deposition with metallic masks is a viable, cost effective method for fabricating binary diffractive optical elements. The method is especially well suited for the infrared region. These diffractive elements are an additional tool for the lens designer. They are of particular value for a system when reduction in weight, cost and size is of interest. The effect of the alignment of the two metallic masks with non-sequential mode in Zemax will be the subject of future publication.

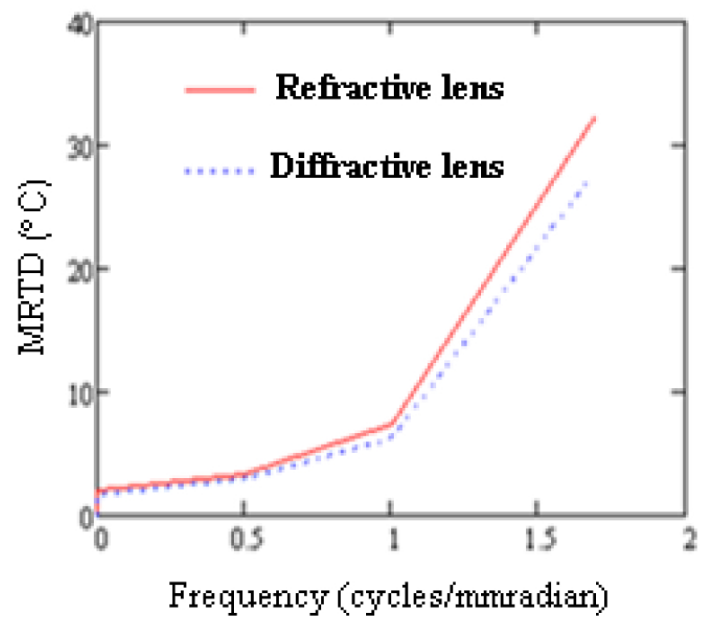

FIG. 9 MRTD curves of refractive/diffractive lenses.

\section{ACKNOWLEDGEMENTS}

This work was totally supported by HIAST (Higher Institute for Applied Sciences and Technology).

The authors gratefully acknowledge the contributions of numerous colleagues in HIAST for their help in the experiments, measurements, and valuable discussions. 


\section{References}

[1] L. Rayleigh, "laboratory notebook," in Physical Optics, R. W. Wood, ed., 37-38 (Macmillan, New York, 1934).

[2] J. Jahns, and S. J. Walker, "Two-dimensional array of diffractive microlenses fabricated by thin film deposition," Appl. Optics 29(7), 931-936 (1990).

[3] H. Dammann, "Blazed Synthetic Phase-Only Holograms," Optic 31, 95-104 (1970).

[4] J. A. Jordan, Jr., P. M. Hirsch, L. B. Lesem, and D. L. Van Rooy, “Kinoform Lenses," Appl. Optics 9, 1883-1887 (1970).

[5] G. J. Swanson, and W. B. Veldkamp, "Diffractive Optical Elements for Use in Infrared Systems," Opt. Eng. 28, 605-608 (1989).

[6] K. Miyamoto, "The Phase Fresnel Lens," J. Opt. Soc. Am. 51, 17-20 (1961).

[7] P. P. Clark, and C. Londofio, "Production of Kinoforms by Single Point Diamond Machining," Opt. News 15, 39-40 (1989).

[8] L. d'Auria, J. P. Huignard, A. M. Roy, and E. Spitz, "Photolithographic Fabrication of Thin Film Lenses," Opt. Commun. 5, 232-235 (1972).

[9] V. P. Koronkevich, "Computer Synthesis of Diffraction Optical Elements," in Optical Processing and Computing, H. H. Arsenault, T. Szoplik, and B. Macukow, eds., 277-313 (Academic Press, Boston, 1989).
[10] G. J. Swanson, Binary Optics Technology (Massachusetts Institute of Technology, Cambridge, 1989).

[11] M. J. Riedl, and J. T. McCann, "Analysis and Performance Limits of Diamond Turned Diffractive Lenses for the 3-5 and 8-12 Micrometer Regions," in Infrared Optical Design and Fabrication, R. Harsman, and W. J. Smith, eds., 153-163 (SPIE Press, Bellingham, 1991).

[12] A. Motogaito, and K. Hiramatsu, "Fabrication of Binary Diffractive Lens on Optical Films by Electron Beam Lithography," in Advances in Unconventional Lithography, G. Kostovski, ed., 139-148 (InTech, Rijeka, 2011).

[13] L. D’Auria, J. P. Huignard, A. M. Roy, and E. Spitz, "Photolithographic Fabrication of Thin Film Lenses," Opt. Commun. 5, 232-235 (1972).

[14] Zemax Product content, http://www.zemax.com.

[15] R. E. Fischer, B. Tadic-Galeb, and P. R. Yoder, Optical System Design (Mcgraw Hill Book, New York City, 2008).

[16] Inframet, The DT 2000G measuring set no 01406 for testing thermal imagers, manual version 2.2. 